\title{
Estado de los repositorios institucionales venezolanos y sus revistas científicas
}

\section{(4) Christian Alexander Martinez-Guerrero y Marisol García Romero \\ Universidad de Los Andes. Escuela de Comunicación Social, Venezuela / camartinezula@gmail.com / Orcid: http://orcid. org/oooo-0oo3-0333-9336 \\ Universidad de Los Andes. Departamento de Español y Literatura, Venezuela / profesoramarisolgarcia@gmail.com / Orcid: http://orcid.org/o0oo-0003-0970-1877}

\section{Resumen}

El Acceso Abierto (AA) se ha convertido en uno de los mecanismos más extendidos dentro de la comunidad científica en el orden mundial. Debido a ello, la presente investigación tuvo como objetivo describir el estado actual de los Repositorios Institucionales (RI) de Venezuela. Además de definir sus procedencias, software y licencias, también se analizaron las publicaciones periódicas alojadas en ellos. Para identificarlos, se tomó como referencia los repositorios digitales catalogados en el Registry of Open Access Repositories (ROAR), luego se determinaron cuáles tenían adscripción universitaria y se desarrollaron listas de cotejo para la recolección de datos de cada uno de los aspectos valorados. Se concretó que en el país hay ocho RI en el ROAR. La mayoría utiliza el DSpace para su desarrollo y alguno de los tipos de licencia Creative Commons; solo en el 50 \% de estos se encuentran almacenadas las revistas científicas de las universidades, de las cuales el 57,2 \% están inactivas o muertas. Ante esto, se recomienda a los gestores y editores científicos crear políticas en beneficio del AA, que garanticen visibilidad en la producción científica de la nación, así como mantener la periodicidad de las publicaciones existentes, pues esto genera mayor calidad.

\footnotetext{
Abstract

State of Venezuelan institutional repositories and their scientific journals. Open Access (OA) has become one of the most expanded mechanisms inside the scientific community around the world. Due to that, this research aimed to describe the current state of the Institutional Repositories (IR) of Venezuela. Apart from defining its origins, software and licenses, periodic publications were also analyzed. To identify them, the digital repositories catalogued in the Registry of Open Access Repositories (ROAR) were taken as a reference, then, it was determined which had university registration and checklists to collect data were used. It was concluded that, in the country, there are eight IR in the ROAR. Most of them use DSpace to their development and some Creative Common licenses; the scientific journals of the universities are present only in $50 \%$ of them, from which the $57.2 \%$ are inactive or dead. Facing this, it is recommended
}

\section{Palabras clave}

Repositorio Acceso abierto Revistas científicas Venezuela

\section{Keywords}

$\overline{\text { Repository }}$ Open access Scientific journals Venezuela 
that scientific managers and editors create policies to benefit the AA that guarantee visibility in scientific production in the country, and maintain the periodicity of the existent publications, as this generates greater quality.

Artículo recibido: 28-08-2017. Aceptado: 26-03-2018

\section{Introducción}

La producción científica, poco tiempo después de su génesis, encontró dos objetivos intrínsecos para su subsistencia: generar una satisfacción (difusión de nuevos saberes destinados a una determinada población) y producir ganancias de esto. La vía para hacer uso de estos contenidos, eventualmente, estuvo ceñida a un definido Acceso Restringido (AR), es decir, solo llegaban a ellos las personas o instituciones que tuvieran la capacidad de pagar. Sin embargo, con el paso de los años, la proliferación de revistas especializadas -consideradas como canales de comunicación académicos respetados mundialmente- $y$, más adelante, la irrupción de la era digital, representó un certero cambio en los modos de consumo. Al respecto, Castells (2003: 4-5) argumenta que

desde el principio, los diseñadores de Internet, todos ellos procedentes del mundo académico (...) buscaron deliberadamente la construcción de una red informática (...). Fue una cultura inspirada en los principios de la investigación académica y en la práctica de compartir los resultados de la investigación.

En consecuencia, desde principios de la década de los 90 empezó a cambiar el panorama (De Volder, 2008). Esto, gracias en gran parte a la invención de las nuevas tecnologías, aquellas cuestionadas condiciones y limitaciones, como las suscripciones lucrativas a las publicaciones periódicas, el impedimento físico para consultar textos considerados como literatura gris -memorias de pre y posgrado, actas de congresos, trabajos de ascensos-, las dificultades para conocer producciones editoriales e incluso las cortapisas idiomáticas han reducido o, cuando menos, se está cada vez más ante una revolución sin precedentes que pretende hacerlo, mediante la puesta en práctica de un connotado mecanismo de oferta: el Acceso Abierto (AA).

El punto de partida de este formato inclusivo encontró su primer antecedente en 1991 cuando se fundaron las revistas científicas Psychology y Surfaces, las cuales dispensaban gratuidad en sus contenidos colgados en la Red, siempre que los usuarios respetaran los derechos de autor (Abadal, 2012). El término se acuñó por primera vez en el año 2002, durante la denominada Budapest Open Access Initiative (BOAI, por sus siglas en inglés). Luego, en Estados Unidos, se produjo el antecedente inicial para el tratamiento específico dirigido a este tipo de producciones intelectuales con la Declaración de Bethesda sobre Publicación de Acceso Abierto. Allí, se avizoraba que el AA sería un elemento fundamental en la producción y publicación científicas, las cuales debían ser, en la mayor medida de lo posible, accesibles y utilizables.

Si bien en esta reunión se alentaba a un mayor compromiso por parte del personal encargado de la difusión de saberes, a finales de 2003, en Alemania, se definieron dos circunstancias esenciales para el avance de esta política: la primera, el aliento al compromiso público por parte de los autores y, además, a la publicación de una versión completa del trabajo en un repositorio digital respaldado por alguna institución, agencia estatal, sociedad de especialistas u organismos pro AA (Berlin Declaration on Open Access to Knowledge in the Sciences and Humanities, 2003).

Esta herramienta tecnológica trata de un dispositivo de almacenamiento de archivos o bases de datos bibliográficas que permiten (1) almacenar los resultados de las 
iniciativas intelectuales de un determinado grupo de personas, (2) aportar visibilidad a estos conocimientos y (3) potenciar la diseminación de estos (Galina, 2011). Pueden distribuirse, de forma intangible, a través de la Red o por medio de un sustento físico, como un CD (García, 2011). Constituyen uno de los servidores más adecuados para el mantenimiento de los estudios, pues no caducan con facilidad, proporcionan enlaces de conexión persistentes y sostienen los documentos aun después de su retiro o desaparición, por lo cual estos forman parte de la denominada vía verde del AA (Suber, 2015).

En sintonía con Abadal (2012), estas herramientas son clasificadas según su objetivo en (1) institucionales: todos aquellos que son llevados a cabo por casas académicas, o (2) temáticos/disciplinares: desarrollados por equipos de investigaciones, instituciones o cualquier otra instancia u organismos de especialización en un área de conocimiento delimitada. No obstante, López (2013) integra a esta tipología tres categorías más: (1) de datos básicos, los cuales son distinguidos por el alojamiento de informaciones elementales para las investigaciones y pueden -o no- coexistir con los institucionales, (2) huérfanos, puestos en marcha para autores que no encuentran cabida en los anteriores espacios digitales explicados y (3) agregadores/recolectores, encargados de agrupar contenidos almacenados en algunos de los dos tipos de repositorios de mayor importancia.

Debido a los escasos antecedentes del tema en el país, este estudio focalizará su interés en ofrecer un diagnóstico y caracterización de los Repositorios Institucionales (RI) ejecutados en el contexto universitario venezolano, suscritos -y constatados por los autores- en el Registry of Open Access Repositories (ROAR), el cual es un directorio realizado por iniciativa de la Universidad de Southampton (Reino Unido) y sostenido desde el 2003, con el software libre y de código abierto, Eprints, que permite la indexación de los repositorios digitales -en cualquiera de sus presentaciones- y que cumplan con la Iniciativa de Archivos Abiertos-Protocolo para la recolección de metadatos (OAI-PMH o Protocolo OAI). Hasta el momento de la investigación (marzo de 2017) en dicho registro se encuentran 4446 espacios virtuales en todo el mundo, los cuales tienen una presencia dominante en países desarrollados, como los EE. UU., y una considerable parte de Europa.

En otro orden, es importante señalar que se definirá la localización -y sus respectivas características- de las revistas científicas en estos RI. Es pertinente aclarar que no se encuentra una data oficial en el país acerca de la cantidad de publicaciones periódicas, pero en el último informe (2016) del Registro de Publicaciones Científicas y Tecnológicas Venezolanas (REVENCYT), de la Universidad de Los Andes (ULA) se reseñan, al menos, 314 .

\section{Materiales y métodos}

Para ofrecer un balance tanto de las revistas científicas alojadas en los RI como del estado actual de cada uno de estos últimos, se consultaron ambas fuentes documentales, de acuerdo con la clasificación de Atkinson (2005), siendo así esta investigación ajustada dentro del paradigma positivista, pues se acercó a la realidad para considerarla y analizarla. La metodología es cuantitativa con complementariedad cualitativa: si bien se manejaron e interpretaron datos numéricos, ellos fueron expuestos a través de la descripción.

En acuerdo con la argumentación de Hernández, Fernández y Baptista (2010), esta investigación, además, cuenta con un diseño no experimental-transversal de tipo descriptivo, ya que la recolección de la información se realizó en un único momento. 
Para el desarrollo del estudio, en primer lugar, se definió la procedencia de los RI, en términos de los tipos de instituciones que los administran - públicas y privadas-, luego, el registro histórico de las fechas de sus creaciones, los tipos de software empleados en estos dispositivos digitales y sus licencias Creative Commons (CC), las cuales, en conformidad con Viñas (2015), nacieron de una Organización No Gubernamental (ONG) sin fines de lucro, de nombre homónima, en EE. UU., y funcionan bajo cuatro condiciones: Atribución, es decir, reconocimiento del autor o los autores por parte de quien haga uso del material; No Comercial; Sin trabajos derivados, por medio de la cual se advierte que, aun cuando haya autorización de publicación, no se permiten modificaciones de la obra; y Compartir bajo condiciones similares, que permite trabajos consecuentes, siempre y cuando se mantenga la identificación inicial. En consecuencia, es posible distinguir seis tipos de licencia CC: Reconocimiento, Reconocimiento - Sin obra derivada, Reconocimiento - Sin obra derivada - No comercial, Reconocimiento - No comercial, Reconocimiento - No comercial - Compartir igual, y Reconocimiento - Compartir igual.

Seguidamente, se tomó los tres criterios formalizados por De Volder (2008: 93), para concretar la etapa de desarrollo en la cual cada uno de ellas se encuentra:

1. Inicial: corresponde al momento de planificación, de la elección del tipo de repositorio, de la demarcación de objetivos, de la definición de los tipos de documentos a ser incluidos, la elección del software, aprobación de las normativas y reglamentaciones correspondientes, etc.

2. Medio: también puede denominarse de prueba o implementación, corresponde al momento de la publicación en línea del repositorio, pero que aún se encuentra como una versión de prueba.

3. Final: corresponde al momento de consolidación del proyecto, luego de la publicación en línea. Esta etapa se evidencia a través del ingreso de documentos en forma continua.

Igualmente, a fin de ofrecer resultados más amplios, se consideraron diferentes criterios de evaluación propuestos por algunos autores (Barrionuevo, 2010; Barrueco, 2014; De Giusti, 2014; Fushimi, Genovés, Pené y Unzurrunzaga, 2011; Serrano, Melero y Abadal, 2014). Para ello, se procedió primero a enviar un cuestionario vía correo electrónico a los administradores de los RI; sin embargo, las respuestas fueron ínfimas, por tanto, se tomaron los propuestos por Barrueco (2014) en la Guía para la elaboración de repositorios institucionales de investigación editada por REBIUN, FECYT y RECOLECTA, los cuales versan predominantemente sobre estos criterios:

"Visibilidad: Se estudia si el repositorio está visible en la propia institución y presente en los directorios nacionales e internacionales. También se refiere a la proporción de documentos del repositorio en acceso abierto.

" Políticas: Los indicadores de este apartado se refieren a si existe una definición de la misión y objetivos del repositorio, y si existe una política pública sobre depósito de documentos, preservación de contenidos y reutilización de metadatos.

"Aspectos legales: Se comprueba si existe una autorización del autor de cada documento introducido en el caso de que el depósito sea mediado, y si está disponible información sobre derechos de autor en el repositorio.

"Metadatos: Estos indicadores van dirigidos a conocer si los propios metadatos siguen una política de indización, y si se permite la exportación de metadatos en algún otro formato que el Dublin Core (Serrano, Melero y Abadal, 2014: 4).

Aun cuando se tratan de 33 criterios, ellos fueron modelados en consonancia con el propósito, las características y la extensión de este estudio. Por añadidura, algunos de 
los ítems prescindidos fueron verificados debido a la propia metodología del trabajo. De esta manera, se hizo especial énfasis en los siguientes:

\section{Políticas}

1. Existe una declaración sobre la misión y objetivos del repositorio.

2. Política pública sobre el archivo en el repositorio, donde se establecen al menos los siguientes puntos: quién puede depositar, qué se puede depositar y en qué formatos.

3. Política pública sobre preservación de los contenidos.

4. Existe una oferta de contacto y asesoramiento visible.

\section{Interoperabilidad}

5. Se marcan los registros eliminados.

\section{Estadísticas}

6. El repositorio proporciona un servicio de estadísticas sobre el uso de los documentos almacenados.

\section{Seguridad}

7. Existe un procedimiento establecido sobre la elaboración de copias de seguridad, tanto del software sobre el que funciona el repositorio, los metadatos y los documentos propiamente dichos.

8. Utilización de identificadores persistentes para sus contenidos (DOIs, Handles, URNs, etc.)

9. Existe una separación de los contenidos de investigación, docencia y cultura que permita a los proveedores de servicios realizar recolecciones selectivas.

Ahora bien, para la selección de los RI analizados, se hizo una exploración de los repositorios venezolanos en línea encontrados en la base de datos de ROAR ${ }^{1}$. En primer lugar, se concretó la cantidad completa de estos instrumentos de preservación científica, los cuales ascienden a la cifra de 21. Posteriormente, se ingresó metódicamente a cada uno de ellos, a fin de verificar sus tipologías, adscripciones institucionales y disponibilidad en la Web.

Más adelante, para la valoración de las publicaciones especializadas allí alojadas, se implementaron indicadores de calidad propuestos por González, Abad, Hernández y Ruiz (2010), que versan sobre el estado en el cual estas se encuentran (Tabla 1). Aunado a ello, se verificó la antigüedad de cada una de ellas y se realizó una clasificación por áreas temáticas.

\begin{tabular}{|l|l|}
\hline Estado & Año de su última publicación \\
\hline Activas & 2017 o 2016 \\
\hline Inactivas & 2015 y no hay notificación sobre su falta de periodicidad \\
\hline Muertas & 2014 o antes, y hay comunicación pública del cese de actividades \\
\hline Sin noticia & No hay más información que la disponibilidad de un único número o volumen \\
\hline
\end{tabular}

Tabla 1. Estado de las re-

Fuente: elaborada a partir de los criterios de González, Abad, Hernández y Ruiz (2010)

\section{Fuentes de la información}

Una vez hecha la exploración, se tuvo una clasificación de ocho RI -38\% del total de la lista- los cuales fueron seleccionados como objetos de estudio, conformando, de esta manera, una muestra de carácter no probabilístico, como reseña Arias (2006).
1. Solo se utilizó como directorio internacional el ROAR y no el OPENDOAR, dado que este último se encuentra desactualizado desde el 2014. Sin embargo, ambos fueron consultados y se cruzaron y describieron los datos para confirmar la presencia de los RI tanto en una fuente como en la otra. Esto demostró, eventualmente, que el ROAR tiene cierto grado de mayor vigencia. Además, los elementos de la muestra también fueron contrastados en el Ranking Web de Repositorios. 
Tabla 2. Procedencia y posicionamiento de los RI venezolanos en $\mathrm{AA}$

\begin{tabular}{|c|c|c|c|}
\hline \multirow[b]{2}{*}{ Repositorio institucional (URL) } & \multirow[b]{2}{*}{$\begin{array}{l}\text { Afiliación } \\
\text { académica }\end{array}$} & \multicolumn{2}{|c|}{$\begin{array}{c}\text { Ranking Web de } \\
\text { Repositorios de } \\
\text { Webometrics (enero } \\
\text { de 2017) }\end{array}$} \\
\hline & & $\begin{array}{c}\text { América } \\
\text { Latina }\end{array}$ & $\begin{array}{l}\text { En el } \\
\text { mundo }\end{array}$ \\
\hline SaberULA (www.saber.ula.ve) & ULA & 4 & 60 \\
\hline Saber UCAB (http://saber.ucab.edu.ve/) & UCAB & 35 & 594 \\
\hline RIUC (http://riuc.bc.uc.edu.ve/) & UC & 105 & 1215 \\
\hline Saber UCV (www.saber.ucv.ve) & UCV & 156 & 1643 \\
\hline Udospace (http://ri.biblioteca.udo.edu.ve/) & UDO & 193 & 1989 \\
\hline $\begin{array}{l}\text { Repositorio institucional de producción } \\
\text { intelectual de la UCLA } \\
\text { (http://repositorio.ucla.edu.ve/) }\end{array}$ & UCLA & 210 & 2187 \\
\hline Miunespace (www.miunespace.une.edu.ve/) & UNE & & \\
\hline Mirai (http://andromeda.unimet.edu.ve/mirai/) & UNIMET & & \\
\hline
\end{tabular}

Fuente: elaboración propia

Los elementos provienen de la ULA, la Universidad Católica Andrés Bello (UCAB), la Universidad de Carabobo (UC), la Universidad Central de Venezuela (UCV), la Universidad de Oriente (UDO), la Universidad Centroccidental Lisandro Alvarado (UCLA), la Universidad de Nueva Esparta (UNE) y la Universidad Metropolitana (UNIMET). Los 13 restantes fueron eximidos de la investigación dado que algunos de ellos no se encontraban en línea, son Bibliotecas Digitales (BD) de instituciones educativas o grupos de investigación y, otros, herramientas electrónicas de empresas privadas, incluso de origen externo al país.

Tres cuartas partes de la muestra -seis de los ocho RI- están también registrados en el OPENDOAR, otro importante directorio de dispositivos digitales en acceso abierto; y también un $75 \%$ de ellos se encuentran posicionados en el Ranking Web de Repositorios llevado a cabo por el Laboratorio de Cibermetría dependiente del Consejo Superior de Investigaciones Científicas (CSIC) y perteneciente al Ministerio de Economía y Competitividad, de España (Tabla 2).

Este informe es desarrollado a partir de cuatro criterios de evaluación: a) tamaño, que versa sobre la cantidad de resultados que arroja el buscador de Google acerca del RI en valoración; b) visibilidad, la cual da cuenta acerca del número total de entradas recibidas provenientes de enlaces externos; $\mathrm{c}$ ) archivos o ficheros, es decir, la suma de documentos alojados en diferentes formatos -pdf, doc, entre otros-, según Google; y d) la cuantía de artículos de un determinado RI recolectados por Google Académico en un período de cinco años. Conviene mencionar que las estimaciones de estos indicadores no son equitativas, pues el segundo de ellos tiene una ponderación del $50 \%$, mientras que, los otros tres, $16,6 \%$, respectivamente (Texier, 2016).

Ahora bien, en acuerdo con la última medición de este reporte (enero de 2017) están posicionados en todo el planeta $2284 \mathrm{RI}$. El $75 \%$ de los ocho correspondientes a la muestra se encuentran allí, pero apenas dos de ellos se ubican en el top 100 de América Latina: SaberULA en el cuarto lugar -solo por detrás de tres pares universitarios brasileros- y Saber UCAB en el renglón número 35. 
El primero de estos dos, conviene resaltar, es el RI con mejor ubicación entre los países de habla hispana procedentes de la región latinoamericana, pues se encuentra en la 60. ${ }^{a}$ plaza. Además, es el cuarto de origen en lengua castellana mejor situado en el mundo, siguiendo solo a Digital CSIC, UPCommons y RUA, todos de España, y localizados en los puestos 30, 32 y 55, respectivamente. En adición, en estos dispositivos se localizaron 159 productos editoriales especializados.

\section{Dificultades para el análisis de los datos}

Antes de presentar los resultados, es necesario mencionar algunas observaciones que se pudieron establecer tanto antes como después de la recolección de datos. En primera instancia, se puntualizó que en la matrícula del ROAR no hay una clara diferenciación entre las BD de acceso abierto de las casas de estudio y los RI, aun cuando hay, como mínimo, cinco connotadas distinciones que las separan: (1) los RI nacen y funcionan en torno a una casa académica, mientras que las BD pueden operar alrededor de una disciplina o cualquier otro aspecto en particular; (2) los RI se manejan a menudo gracias a la disposición del autoarchivo que usan los autores, las $\mathrm{BD}$, no; (3) en el caso de las $\mathrm{BD}$, se puede contar con servicios extras (de personal, de apoyo, de referencias) que normalmente los RI no lo tienen; (4) estos últimos, a su vez, se desarrollan bajo un sistema informático predeterminado, las BD, no tienen esa obligación; y (5) en la mayoría de las ocasiones, los archivos de los RI son de origen digital y los de las $\mathrm{BD}$, aunque también gocen de este privilegio, suelen ser documentos digitalizados (Texier, 2013).

Del mismo modo, conviene aclarar que, en algunos centros educativos venezolanos, las revistas científicas reposan en dichas $\mathrm{BD}$-como es el caso de la Universidad Nacional Abierta (UNA) o algunos de los medios especializados de la Universidad Simón Bolívar (USB) (2011)-, sin embargo, tal cual explican Babini, González, López y Medici (2010), son los RI las herramientas idóneas para la proyección de la imagen académica, ya que en estos se guarda la producción científica desarrollada por el personal intelectual adscrito a una $u$ otra universidad.

En otro orden de ideas, como se esbozó arriba, solo una minoría de los gestores de los RI retribuyó el contacto comunicacional con los investigadores. No obstante, vale aclarar que Rafael Navea, administrador de Mirai, argumenta (correo electrónico, 19 de febrero de 2017) que este "no es totalmente un repositorio porque no todos los registros tienen acceso al texto completo, por eso se llama Módulo de Información y Repositorio". Dicha particularidad se ve reflejada en que sus tres revistas científicas (Cuadernos Unimetanos, Anales y Alamanaque) se hallan solo en el servidor principal de la universidad, dentro de su apartado de publicaciones.

\section{Análisis y resultados}

\section{Repositorios institucionales venezolanos}

La Oficina de Planificación del Sector Universitario (OPSU) es la institución venezolana encargada de garantizar la gestión administrativa nacional de las universidad, sin embargo, en sus plataformas digitales no se registran cifras oficiales sobre la cantidad -y procedencia: pública o privada- del total de casas de estudios superiores en la nación. En el sitio web del Ministerio del Poder Popular para la Educación Universitaria, Ciencia y Tecnología sí hay un directorio de instituciones educativas, mas no está completo, porque se descubrió allí la falta de información de las universidades privadas. 
Figura 1. Universidades venezolanas con RI de AA

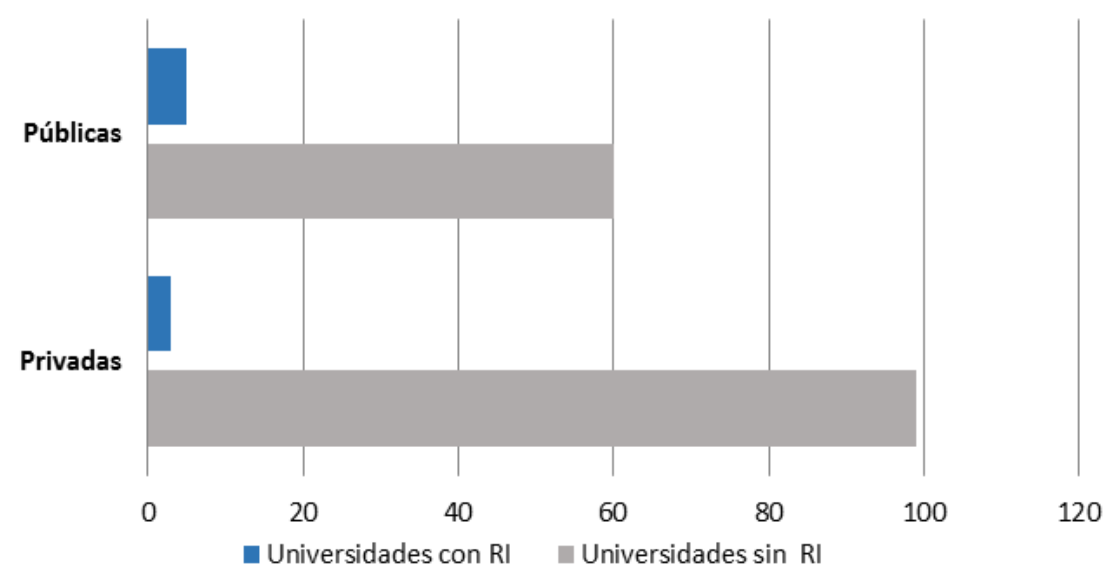

Fuente: elaboración propia

No obstante, en afinidad con un informe elaborado por la USB (2011), hay un total de 60 universidades públicas y 99 privadas. Así, una vez procesados los datos, se determinó en primer lugar que, en contraste con el directorio del ROAR, en solo el $4,8 \%$ de ellas cuenta con un dispositivo digital de tal magnitud para el almacenamiento de sus resultados de investigaciones. De esta cifra, el 62,5\% de los RI proviene de universidades públicas -ULA, UCV, UDO, UC y UCLA-; y 37,5\% de universidades privadas-UCAB, UNIMET y UNE (Figura 1).

En conformidad con la información del ROAR, acerca del registro histórico de estos RI, en el país datan desde el año 2000, con la invención de SaberULA, pero se originó una pausa de una década para que hubiese otra iniciativa universitaria de este tipo: no fue sino hasta el 2011 cuando fueron puestos en marcha Saber UCAB, Miunespace y Udospace; en el año siguiente, el crecimiento fue sostenido mediante el desarrollo del RIUC, Saber UCV y el Repositorio institucional de producción intelectual de la UCLA. Finalmente, en 2013, se originó Mirai. Desde allí, hasta la fecha del estudio (primer trimestre de 2017) no se han elaborado más, al menos, para el área de cobertura de este directorio.

Por otro lado, los repositorios digitales, por su naturaleza tecnológica y sus múltiples tareas de funcionabilidad, deben requerir la utilización de un tipo de software que permita su administración en acuerdo con las exigencias y normativas de sus hacedores. DSpace, gracias a sus características de licencia libre, la garantía del acceso abierto y su gratuidad, es ubicado como el conjunto de programas y herramientas informáticas más usado en el mundo para la práctica de esta actividad (Figura 2).

En cuanto a los tipos de software usados por los elementos de la muestra se notó una tendencia similar, puesto que siete de ellos, funcionan con la aplicación de DSpace, mientras que solo uno, Mirai, opera con WinIsis.

Ahora bien, sobre los tipos de licencia CC bajo los cuales se norman los RI analizados, se conoció que SaberULA y Saber UCAB operan bajo la licencia Reconocimiento - No comercial - Compartir igual; mientras que Saber UCV y Mirai tienen su garantía de CC de carácter Reconocimiento - Sin obra derivada - No comercial.

El RIUC, aunque en su descripción asegura funcionar con alguna de estas licencias, más abajo declara que sus obras allí alojadas pueden ser comercializadas, lo cual no está enmarcado en alguna de las seis licencias CC. En Miunespace y en el Repositorio institucional de producción intelectual de la UCLA no hay acceso directo a esta 


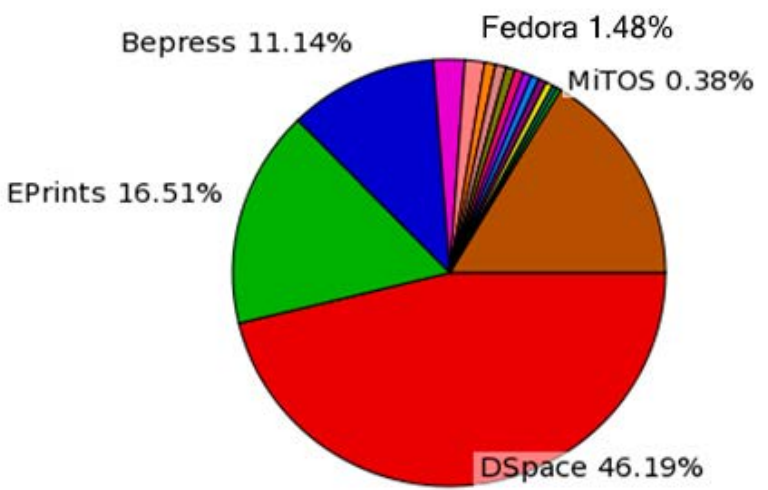

DSpace Bepress Fedora

ETD-db

DIGIBIB

CDS Invenio

Open Repository

Fedora $>$ Fez

Fuente: ROAR (2017)

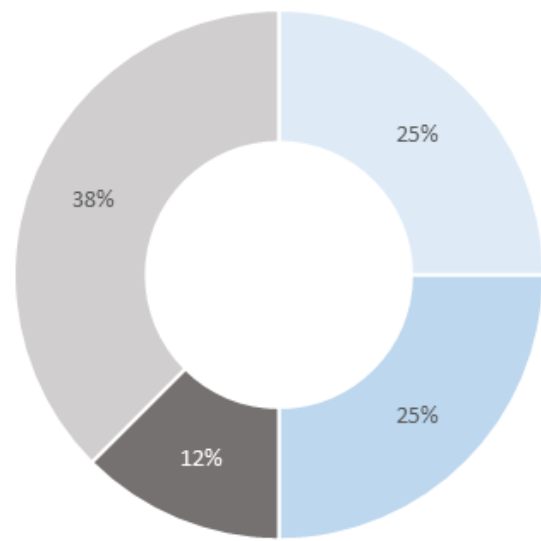

U CC Reconocimiento- No

comercial - Compartir igual

w CC Reconocimiento-Sin obra derivada - No comercial

- RI ana lizados sin aplicación de licencias CC

- $\mathrm{RI}$ a na lizados sin descripción de

licencias de derechos de autor

Fuente: elaboración propia

información, ni fue contestada la solicitud de comunicación por parte de sus gestores (Figura 3).

En cuanto a la etapa de desarrollo en la cual está cada uno de los RI analizados, se diagnosticó que, aun cuando en todos ellos se puede acceder en mayor o menor medida a los documentos, solo cuatro podrían ser encasillados dentro de la etapa final del proyecto universitario: SaberULA, Saber UCAB, Saber UCV y el RIUC. Los cuatro restantes muestran evidentes falencias en términos de facilidad, agilidad y recuperación de contenidos, por tanto, su nivel de desarrollo es medio, en correspondencia con las fases descritas anteriormente.

Con respecto a su evaluación, en consonancia con la Guía para la elaboración de repositorios institucionales de investigación, se detectaron deficiencias sobre todo en cuanto a sus políticas, su interoperabilidad, estadísticas y seguridad. Así, ninguno de los RI tiene declarada su misión y solo tres muy someramente sus objetivos. Solo uno,
Figura 2. Tipos de software más utilizados en los repositorios digitales

Figura 3. Aplicación de licencias $\mathrm{CC}$ en los RI venezolanos de AA 
Figura 4. Estado de las revistas científicas alojadas en los RI venezolanos en AA

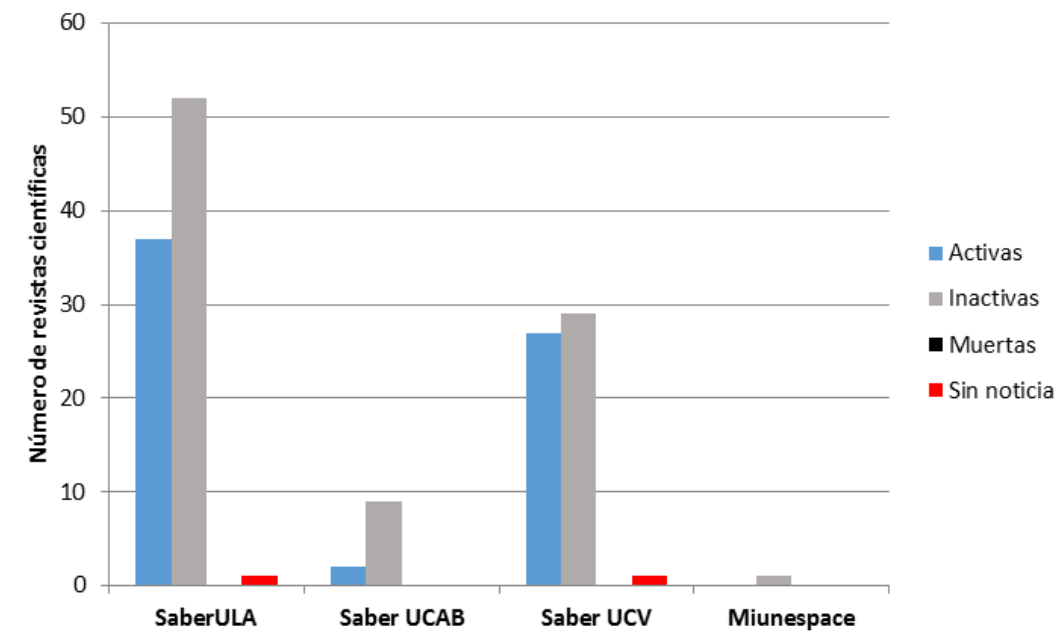

Fuente: elaboración propia

SaberULA, establece quién puede depositar, en cuáles formatos y qué tipo de contenidos. De igual modo, aunque en todos los elementos de la muestra hay una oferta de contacto para posibles asesoramientos a autores, se percibió que el índice de respuesta es bajo.

Los registros o archivos eliminados no son marcados en ninguno de estos RI, ni tampoco se encuentra disponible los lineamientos de tiempo de preservación o supresión de los documentos. Y se constata el mismo escenario acerca de la disponibilidad de un servicio de estadísticas, tanto de los documentos almacenados como de sus consultas; al igual que en lo referente al uso de identificadores persistentes -como el Digital Object Identifier (DOI)-, al procedimiento para el fomento de copias de seguridad del software y los documentos alojados en los RI. Y tampoco se halla una segregación de los contenidos o disciplinas abordadas, lo cual no permite llevar a cabo recolecciones selectivas.

\section{Los repositorios institucionales venezolanos como mecanismo de visibilidad para las revistas científicas}

Una vez revisados los elementos de la muestra, se concretó que el $50 \%$ de estas ocho universidades alojan sus revistas científicas en sus RI. Esta conducta se deriva específicamente de la ULA, la UCAB, la UCV y la UNE. Cabe precisar que, en el caso de esta última, aunque se editan tres revistas científicas, se conoció que en su RI, Miunespace, apenas se localiza una, mientras que las dos restantes reposan en otros servidores en línea distintos.

Bajo tales condiciones descritas, se encontraron 159 medios especializados en los cuatro dispositivos electrónicos: 90 de SaberULA, 11 de Saber UCAB, 57 de Saber UCV y uno de Miunespace. En el primero de ellos hay 37 activas, 52 inactivas y una sin noticia; en la otra universidad pública, 27, 29 y una, respectivamente. En la $\mathrm{UCAB}$, dos activas y nueve inactivas; y en la UNE, una sola, inactiva (Figura 4).

En cuanto a la antigüedad de estas 159 publicaciones periódicas, se descubrió que en poco más de la mitad ellas $\left(51,6 \%-84^{-}\right)$no se hace alusión al año en las cuales fueron creadas o adaptadas al formato digital. Por ende, con los datos disponibles, se observó que la década de los 90 fue la más productiva -en cuanto a emprendimiento 


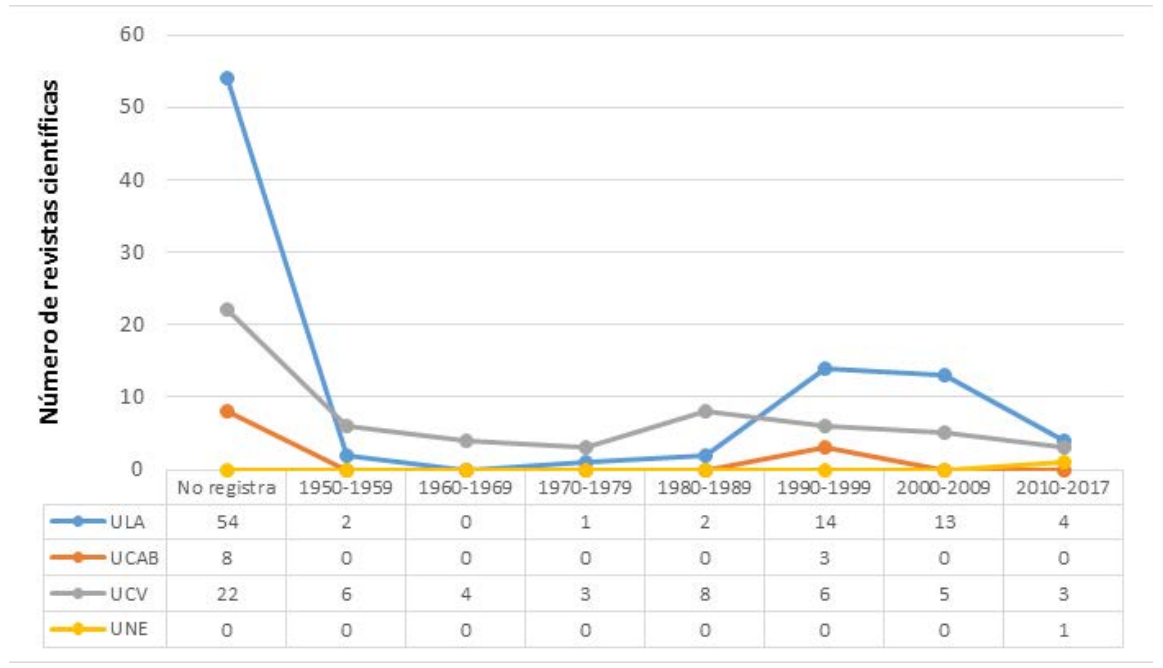

Fuente: elaboración propia

editorial-, pues iniciaron actividad 23 medios especializados procedentes de estas cuatro instituciones universitarias. En orden decreciente, entonces, vieron luz pública 18 revistas científicas durante los primeros diez años del nuevo milenio, otras 10 en los años 80, ocho más desde el 2010 hasta el primer trimestre del 2017, ocho en la década de los 50 del pasado siglo XX, además de cuatro por cada uno de los 10 años siguientes, es decir, en los 60 y 70 (Figura 5).

Acerca de las áreas del conocimiento a las cuales están dirigidos estos medios especializados, se empleó la Clasificación Unesco de las áreas de ciencia y tecnología, y se concretó que hay un predominio en las líneas editoriales que versan sobre estudios interdisciplinarios, seguidos por la amplia área temática de la medicina y biomedicina, las teorías y métodos educativos y las teorías, análisis y críticas literarias, debido fundamentalmente a la tendencia hacia las ciencias sociales o humanas trazada por los comités editoriales de las revistas científicas de la ULA y la UCV. Por tanto, es importante señalar que las mencionadas políticas editoriales representan un 18,$23 ; 15,09 ; 10,69$ y $6,91 \%$, respectivamente, de la ocupación total del tratamiento de las 159 publicaciones periódicas. Así, se observó una marcada desproporción, pues mientras estas cuatro líneas de investigación ocupan una mayoría simple del $51 \%$ de las áreas temáticas, poco menos de la mitad restante aglutina 26 áreas de conocimientos, entre las cuales destacan los estudios culturales, históricos, geográficos, de la agricultura o agronomía, la ingeniería, la comunicación, entre otros (Anexo 1).

De esta manera, se puntualizó que, dejando de lado las revistas con orientación interdisciplinaria mayormente de temas sociales y, utilizando el patrón de clasificación, las Ciencias Médicas es el bloque de estudio con mayor penetración en la muestra académica venezolana aquí analizada -con 27 revistas científicas-. Luego, se ubica, respectivamente, la Pedagogía -con 17-, las Ciencias Agrarias -con 13-, la Sociología y las Ciencias de las Artes y las Letras -con 10, por cada lado-, las Ciencias Tecnológicas -con ocho-, las Ciencias Económicas, Geografía, Historia y Filosofía -con seis, cada una de ellas-, las Ciencias Políticas -con cuatro-, las Ciencias Jurídicas y Derecho, y las Ciencias de Vida -con tres, equitativamente-, las Ciencias de la Tierra y el Espacio, la Antropología, y la Psicología -con dos, para un total de seis entre las tres-, y la Química y la Biología -con una, por cada caso-.
Figura 5. Registro histórico de las revistas científicas alojadas en los RI venezolanos en AA 


\section{Discusión y conclusiones}

Expuestos los resultados, se puede resaltar que las iniciativas universitarias en Venezuela con respecto al AA de la ciencia, específicamente, bajo el apoyo de repositorios institucionales, son escasas, si se tiene en cuenta la cantidad de casas de estudios superiores y la exigua muestra de ellas que cuentan con estas herramientas digitales. Dicha situación podría atribuirse a la falta de regularización por parte de los entes nacionales gubernamentales y académicos correspondientes, lo cual contrasta acentuadamente con la realidad de otros países de la región, citaremos algunos casos: la Ley N. ${ }^{\circ}$ 26899: Creación de Repositorios Digitales Institucionales de Acceso Abierto, Propios o Compartidos (en Argentina), los Lineamientos Generales para el Repositorio Nacional y los Repositorios Institucionales, expedidos por el Consejo Nacional de Ciencia y Tecnología (CONICYT), a partir de la Ley de Ciencia y Tecnología (en México) o la Ley N. ${ }^{\circ} 30035$ que Regula el Repositorio Nacional Digital de Ciencia, Tecnología e Innovación de Acceso Abierto (en Perú).

En consecuencia, este es un cambio necesario que debe proceder desde el marco legal de las instancias gubernamentales del país. Entretanto, se recomienda a los gestores científicos de la academia venezolana que no solo creen nuevos RI en AA, sino también que mantengan y potencien los ya existentes, en pro de la socialización de las investigaciones bajo el rango de la administración, la preservación y reutilización de los contenidos, la denotación de calidad de las instituciones y la comodidad para el fomento de las publicaciones electrónicas que ofrecen los RI (Calderón-Martínez y Ruiz-Conde, 2013; Cano, De Dios, García y Cuesta, 2010; Hallo y De la Fuente, 2010).

Del mismo modo, a las universidades y administradores de los RI de la UDO, UCLA, RIUC y Mirai, se sugiere depositar las publicaciones periódicas en estas herramientas digitales, ya que esto se encuentra entre sus funciones y, además, ayuda a potenciar la visibilidad de las investigaciones (Barruecos y García 2009). Conviene también que los administradores de los ocho objetos de estudio en general registren o mejoren en ellos su información básica acerca de sus reseñas históricas y sus aspectos relativos a las políticas $\mathrm{y}$ funcionamiento para el abordaje de futuras investigaciones sobre RI.

Por otro lado, durante el estudio se descubrió que la casa de estudios venezolana con mayor producción científica de Venezuela, según los indicadores cienciométricos de la Red de Revistas Científicas de América Latina y el Caribe, España y Portugal_(REDALYC, 2017), La Universidad del Zulia (LUZ), ha venido desarrollando su RI desde el 2013, pero aún no se encuentra disponible en la Red, por ello, sus 30 revistas científicas todavía permanecen en un servidor que funciona bajo el Open Journal System (OJS) y se ubica con un enlace directo en su sitio web principal.

En cuanto a los medios especializados alojados en la muestra del estudio, se precisó que 1) una porción considerable de ellos destina sus políticas editoriales a disciplinas vinculadas con las ciencias sociales o humanísticas, lo cual muestra un desequilibrio en contraste con las pertenecientes a las ciencias del área tecnológica. 2) La mayoría de ellos no ofrecen reseñas relativas al año de creación, las etapas por las cuales han atravesado o el momento en el cual comenzaron su marcha en línea, lo cual constituye una falla en la gestión editorial, pues este dato es tomado en cuenta por algunas instituciones para procesos de indexación. 3) Se evidencia una marcada tendencia hacia la inactividad, a saber, no hay entregas de números de los productos editoriales desde el 20150 antes. Este descubrimiento coincide con la reflexión de Pérez (2013: s/p):

la periodicidad, como criterio de calidad e impacto de las revistas científicas, desde el año 2009, dejó de cumplirse, debido principalmente a la falta de presupuesto, a la demora en la entrega de los recursos correspondientes, o a la no asignación de estos. 
No obstante, en la gran mayoría de estos medios especializados tampoco se dispone información pública sobre sus posibles muertes o las razones que fomentan el incumplimiento de sus apariciones, lo cual deja ver otra deficiencia en la gestión editorial. De este modo, se propone a sus directores o editores hacer una comunicación pública acerca del estado por el cual atraviesan y dejar constancia sobre su discontinuidad o receso, puesto que despejaría dudas de los autores y lectores en cuanto a la falta de los nuevos números.

Esta inactividad podría atribuirse a razones presupuestarias en las universidades públicas. Ello, en parte, gracias a que desde el 2001, año en el que fue creado el Fondo Nacional de Ciencia y Tecnología (FONACIT), ente encargado de garantizar y administrar el financiamiento de las investigaciones en Venezuela, los recursos destinados a la ciencia mermaron en un 80 \% (Fermín, 2016). A la par, desde el 2005 con la promulgación de la Ley Orgánica de Ciencia, Tecnología e Investigación (Locti), se permitieron intercambios entre la empresa privada y la academia, aunque cinco años más tarde las condiciones cambiaron y el aporte "ya no podía ir directamente a los investigadores sino a un fondo, cuyos recursos son distribuidos por el gobierno" (San Blas, 2017, citado en Muñoz, 2017: 1).

Empero, también denota, como sostienen Pereira, Casanova y Pire (2014: 75):

una realidad a la que subyace la productividad científica en las universidades privadas [la cual es] la carencia de procesos que promuevan la transferencia de conocimiento (...) para dar validez y confiabilidad a las líneas de investigación de estas instituciones.

En otras palabras, se percibe una mayor relevancia a las actividades de docencia que a las de investigación y extensión. Tal situación ha generado consecuencias graves. En cifras, en afinidad con un estudio de la revista científica Nature, Venezuela fue el único país de América del Sur que sufrió un descenso cuantitativo en cuanto a la investigación especializada. Y aunque estadísticamente ha crecido el número de autores certificados -25000 en total, cifra elevada en comparación con los pares de la región- no así su contribución directa en términos de resultados de innovación e investigación (Ramírez y Salcedo, 2016). A esto, además, se le debe agregar que, durante los últimos 15 años, más de 1500 científicos han emigrado de la nación (Gutiérrez, 2016). 


\section{Anexo 1}

Áreas temáticas de las revistas científicas, según la clasificación de la Unesco, alojadas en los RI venezolanos en AA

Fuente: elaboración propia

\begin{tabular}{|c|c|c|c|c|c|c|}
\hline Área temática & SaberULA & $\begin{array}{l}\text { Saber } \\
\text { UCAB }\end{array}$ & Saber UCV & Miunespace & Total & $\%$ \\
\hline Interdisciplinarias & 13 & 3 & 12 & 1 & 29 & 18,23 \\
\hline Medicina/Biomedicina & 9 & & 15 & & 24 & 15,09 \\
\hline Teorías y métodos educativos & 13 & 2 & 2 & & 17 & 10,69 \\
\hline Teorías, análisis y críticas literarias & 7 & & 4 & & 11 & 6,91 \\
\hline Agricultura/Agronomía & 5 & & 2 & & 7 & 4,40 \\
\hline Historia general & 5 & & 1 & & 6 & 3,77 \\
\hline Geografía & 3 & & 3 & & 6 & 3,77 \\
\hline Filosofía & 3 & 1 & 2 & & 6 & 3,77 \\
\hline Peces y fauna silvestre & 4 & & 1 & & 5 & 3,14 \\
\hline Arte/Cultura & 4 & & & & 4 & 2,51 \\
\hline Ingeniería & 1 & 1 & 2 & & 4 & 2,51 \\
\hline Comunicación/Periodismo & 1 & 1 & 1 & & 3 & 1,88 \\
\hline Estudios de género & 2 & & 1 & & 3 & 1,88 \\
\hline Farmacología & 1 & & 2 & & 3 & 1,88 \\
\hline Procesos tecnológicos & 1 & & 2 & & 3 & 1,88 \\
\hline Derecho & 3 & & & & 3 & 1,88 \\
\hline Organización y dirección de empresas & 1 & 1 & 1 & & 3 & 1,88 \\
\hline Geopolítica & 2 & & & & 2 & 1,25 \\
\hline Antropología social & 2 & & & & 2 & 1,25 \\
\hline $\begin{array}{l}\text { Organización industrial y políticas } \\
\text { gubernamentales }\end{array}$ & 1 & 1 & & & 2 & 1,25 \\
\hline Criminología & 1 & & 1 & & 2 & 1,25 \\
\hline Economía general & 2 & & & & 2 & 1,25 \\
\hline Arquitectura & 1 & & 1 & & 2 & 1,25 \\
\hline Botánica & & & 2 & & 2 & 1,25 \\
\hline Psicología general & & 1 & 1 & & 2 & 1,25 \\
\hline Diseño & 1 & & . & & 1 & 0,62 \\
\hline Biología & & & 1 & & 1 & 0,62 \\
\hline Ecología & 1 & & & & 1 & 0,62 \\
\hline Química analítica & 1 & & & & 1 & 0,62 \\
\hline Contabilidad económica & 1 & & & & 1 & 0,62 \\
\hline Ciencias veterinarias & 1 & & & & 1 & 0,62 \\
\hline Total & 90 & 11 & 57 & 1 & 159 & 100 \\
\hline
\end{tabular}




\section{Referencias Bibliográficas}

》Abadal, Ernest. 2012. Acceso abierto a la ciencia. Barcelona: Editorial UOC.

" Arias, Fidias. 2006. El proyecto de investigación. Introducción a la metodología científica. $4^{\circ}$ ed. Caracas: Episteme.

》Atkinson, Paul. 2005. Qualitative research - Unity and diversity. En Forum. Vol. 6, no. 3, 32-47.

»Babini, Dominique; Jessica González; Fernando López y Flavia Medici. 2010. Construcción social de Repositorios Institucionales: el caso de un repositorio de América Latina y el Caribe. En Información, cultura y sociedad. No. 23, 63-90.

»Barrionuevo, Leticia. 2010. Indicadores de calidad para evaluar repositorios institucionales. <http://hdl.handle.net/10251/8672> [Consulta: 10 abril 2018].

"Barrueco, José y Cristina García. 2009. Repositorios institucionales universitarios: evolución y perspectivas. En XI Jornadas Españolas de Documentación (1995: Zaragoza). Trabajos presentados. Zaragoza: Interinformación. p. 99-108.

"Barrueco, José. 2014. Guía para la evaluación de repositorios institucionales de investigación. Madrid: FECYT, RECOLECTA y CRUE. <http://digital.csic.es/bitstream/10261/35735/1/GuiaEvaluacionRecolectav1.0-1.pdf> [Consulta: 6 abril 2018].

"Berlin Declaration on Open Access to Knowledge in the Sciences and Humanities. 2003. <http://oa.mpg.de/openaccess-berlin/berlindecla ration.html> [Consulta: 21 marzo 2008].

» Budapest Open Access Initiative. 2002. <http://www.soros.org/openaccess/ read.shtml> [Consulta: 20 marzo 2017].

»Calderón-Martínez, Aurora y Enar Ruiz-Conde. 2013. Participación y visibilidad web de los repositorios digitales universitarios en el contexto europeo. En Comunicar. Vol. 20, no. 40, 193-201.

»Cano, Anisley; Raiza De Dios; Ognara García y Floriselda Cuesta. 2010. Los repositorios institucionales: situación actual a nivel internacional, latinoamericano y en Cuba. En Revista Cubana de Información en Ciencias de la Salud. Vol. 26, no. 4, 314-329.

》Castells, Manuel. 2003. Internet, libertad y sociedad: una perspectiva analítica. En Polis. No. 4, 1-20.

"De Giusti, Marisa. 2014. Una metodología de evaluación de repositorios digitales para asegurar la preservación en el tiempo y el acceso a los contenidos (Tesis doctoral). Universidad Nacional de La Plata, Buenos Aires, Argentina. <http:// sedici.unlp.edu.ar/handle/10915/43157> [Consulta: 10 abril 2018].

»De Volder, Carolina. 2008. Los repositorios de acceso abierto en la argentina. Situación actual. En Información, Cultura y Sociedad. No. 19, 79-98.

》Fermín, M. 2016. Venezuela retrocedió en producción científica. En El Nacional. <https://goo.gl/VHtOSb> [Consulta: 28 marzo 2017].

» Fushimi, Marcela; Patricia Genovés; Mónica Pené y Carolina Unzurrunzaga. 2011. Indicadores para evaluar repositorios universitarios argentinos, de la teoría a la práctica. <http://www.memoria.fahce.unlp.edu.ar/trab_eventos/ev.878/ ev.878.pdf> [Consulta: 6 abril 2018]. 
»Galina, Isabel. 2011. La visibilidad de los recursos académicos. Una revisión crítica del papel de los repositorios institucionales y el acceso abierto. En Investigación Bibliotecológica. Vol. 25, no. 53, 159-183.

» García, Jesús. 2011. Desarrollo y uso de repositorios institucionales en las bibliotecas académicas de América Latina y el Caribe. En Martínez, F., coord., Acceso Abierto a la información en las Bibliotecas Académicas de América Latina y el Caribe. México: Universidad Nacional Autónoma de México, Centro Universitario de Investigaciones Bibliotecológica. p. 71-82.

»González, Aurora; María Abad; Javier Hernández y Julia Ruiz. 2010. Localización e identificación de las revistas universitarias para su integración en repositorios institucionales. El caso de la Universitat de València. En BID. No. 25. <http://mobiroderic.uv.es/bitstream/handle/10550/25423/localizacio_identificacion_revistas_UV.pdf?sequence $=1 \&$ isAllowed $=y>$ [Consulta: 31 marzo 2017].

» Gutiérrez, Alejandro. 2016. La investigación e innovación hoy en Venezuela. En Revista Venezolana de Endocrinología y Metabolismo. Vol. 14, no. 3, 166-167.

» Hallo, María y Pablo De la Fuente. 2010. Preservación digital en el contexto de repositorios institucionales universitarios. En Revista Cubana de Ciencias Informáticas. Vol. 4, no. 3 y 4, 1-15.

»Hernández, Roberto; Carlos Fernández y Pilar Baptista. 2010. Metodología de la investigación. $5^{\circ}$ ed. México: McGraw-Hill Interamericana.

"López, Fernando Ariel. 2013. Visibilidad e impacto de los repositorios digitales en acceso abierto. En De bibliotecas y bibliotecarios... Boletín Electrónico Abgra. No. 5, 1-12.

»Muñoz, Fernando. 2017. Descenso en la investigación científica marca centenario de la Academia de Ciencias de Venezuela. En NotiGlobal. <http://notiglobal.com.ve/index.php?option=com_k2\&view=item\&id=18790: descenso-enla-investigacion-cientifica-marca-centenario-de-la-academia-de-ciencias-devenezuela\&ltemid=120> [Consulta: 30 marzo 2017].

»Pereira, Aracelys; Mery Casanova y Reinaldo Pire. 2014. Estudio comparativo de la producción científica de las universidades públicas y privadas venezolanas. En Compendium. No. 32, 55-77.

»Pérez, Josefa. 2013. Statu quo de las revistas científicas de la Universidad Pedagógica Experimental Libertador e impacto del Atlas Cienciométrico de Venezuela y del Sistema Abierto de Revistas. En Letras. Vol. 55, no. 88, 12-45.

»Ramírez, Tulio y Audy Salcedo. 2016. Inversión y producción científica en Venezuela. ¿Una relación inversamente proporcional? En Revista de Pedagogía. Vol. 37, no. 101, 147-174.

»Red de Revistas Científicas de América Latina y el Caribe, España y Portugal. REDALYC. 2017. Indicadores cienciométricos 2005-2014. <http://www.redalyc. org/pais.oa?id=94\&tipo=indicadores\&perfil=produccion $>$ [Consulta: $31 \mathrm{mar}-$ zo 2017].

"Serrano, Rocío; Remedios Melero y Ernest Abadal. 2014. Indicadores para la evaluación de repositorios institucionales de acceso abierto. En Anales de Documentación. Vol. 17, no. 2, 1-12.

"Suber, Peter. 2015. Acceso abierto. México: Universidad Autónoma del Estado de México: Colección Cuadernos Institucionales.

» Texier, José. 2013. Los repositorios institucionales y las bibliotecas digitales: una somera revisión bibliográfica y su relación en la educación superior. En 
$11^{\circ}$ Latin American and Caribbean Conference for Engineering and Technology (2013: Cancún). Trabajos presentados. Cancún. <http://eprints.rclis.org/19925/1/ LACCEI\%202013\%20-\%20Texier.pdf> [Consulta: 31 marzo 2017].

" Texier, José. 2016. Los repositorios institucionales y su importancia en las universidades venezolanas. En Scitus. No. 1, 81-90.

"Universidad Simón Bolívar. 2011. Sistema de Educación Superior en la República Bolivariana de Venezuela. <http://www.telescopivzla.net/docs/sistema_educacion_2011.pdf> [Consulta: 29 marzo 2017].

»Viñas, Mariela. 2015. El uso de licencias Creative Commons en las bibliotecas universitarias argentinas. En Questión. Vol. 1, no. 47, 449-472. 
